\title{
Clinical and microbiological characteristics of tigecycline non-susceptible Klebsiella pneumoniae bacteremia in Taiwan
}

\author{
Yi-Tsung Lin ${ }^{1,2^{*}}$, Fu-Der Wang ${ }^{1,2}$, Yu-Jiun Chan ${ }^{1,2,3}$, Yung-Chieh Fu ${ }^{3}$ and Chang-Phone Fung ${ }^{1,2}$
}

\begin{abstract}
Background: Resistance among Klebsiella pneumoniae to most antibiotics is on the rise. Tigecycline has been considered as one of the few therapeutic options available to treat multidrug-resistant bacteria. We investigated the clinical and microbiological characteristics of tigecycline non-susceptible K. pneumoniae bacteremia.

Methods: Adult patients with tigecycline non-susceptible K. pneumoniae bacteremia at a medical center in Taiwan over a 3-year period were enrolled. K. pneumoniae isolates were identified by the E-test using criteria set by the US Food and Drug Administration (FDA). Data on the clinical features of patients were collected from medical records. Genes for $\beta$-lactamases, antimicrobial susceptibilities and pulsed-field gel electrophoresis (PFGE) results were determined for all isolates.

Results: Of 36 patients, 27 had nosocomial bacteremia. Overall 28-day mortality was $38.9 \%$. The $\mathrm{MIC}_{50}$ and $\mathrm{MIC}_{90}$ of tigecycline were 6 and $8 \mathrm{mg} / \mathrm{L}$, respectively. No carbapenemase was detected among the 36 isolates. Twenty isolates carried extended spectrum $\beta$-lactamases and/or DHA-1 genes. No major cluster of isolates was found among the 36 isolates by PFGE. Intensive care unit onset of tigecycline non-susceptible Klebsiella pneumoniae bacteremia was the only independent risk factor for 28-day mortality.

Conclusions: The high mortality of patients with tigecycline non-susceptible $K$. pneumoniae bacteremia may suggest a critical problem. Further study to identify the possible risk factors for its development and further investigation of this type of bacteremia is necessary.
\end{abstract}

Keywords: Antimicrobial resistance, Bacteremia, Clinical characteristics, Klebsiella pneumoniae, Tigecycline

\section{Background}

Klebsiella pneumoniae is a common cause of communityacquired and hospital-acquired Gram-negative infection of the bloodstream [1]. The vast majority of $K$. pneumoniae infections (including urinary-tract infections, pneumonia, and intra-abdominal infections) are associated with hospitalization [2]. In Taiwan, K. pneumoniae is also a major cause of community-acquired pyogenic infection [3-7].

$K$. pneumoniae strains harboring extended spectrum $\beta$-lactamases (ESBL) and, more recently, carbapenemase that confer resistance to multiple antibiotics, have been described in many parts of the world. These multidrug-

\footnotetext{
*Correspondence: ytlin8@vghtpe.gov.tw

'Division of Infectious Disease, Department of Medicine, Taipei Veterans General Hospital, No. 201, Sec. 2, Shih-Pai Road, Taipei 112, Taiwan

${ }^{2}$ School of Medicine, National Yang-Ming University, Taipei, Taiwan Full list of author information is available at the end of the article
}

resistant (MDR) organisms affect the choice of antimicrobial therapy, and are a major cause for increasing hospital costs and duration of hospitalizations [8].

Tigecycline is an expanded broad-spectrum antibiotic representing a new class called "glycylcyclines". In comparison with other tetracyclines, tigecycline displays higher in vitro activity against several Gram-positive and various Gram-negative microorganisms (including MDR strains). Resistance among $K$. pneumoniae to most antibiotics is on the rise globally [9]. However, tigecycline remains active against many strains, and regarded as one of the few therapeutic options available for treating MDR bacteria.

Tigecycline resistance in K. pneumoniae has increasingly been reported in European countries, with a non-susceptible prevalence of 7.5-50\% [10]. Reports from North America, South America and Asia have demonstrated that the 
non-susceptible prevalence is $<10 \%$ [10]. The increasing prevalence of tigecycline resistance is a growing concern clinically. However, reports of patients infected with tigecycline- resistant $K$. pneumoniae have rarely been identified [11-14]. Furthermore, no study has systematically analyzed clinical data on tigecycline non-susceptible isolates causing bloodstream infection in adult patients.

We investigated the clinical and microbiological characteristics of bacteremia caused by tigecycline non-susceptible K. pneumoniae in adult patients during 2010-2012 from a medical center in Taiwan.

\section{Methods}

\section{Study population}

This retrospective study was conducted at Taipei Veterans General Hospital (a 2900-bed tertiary-care teaching hospital) from January 2010 to December 2012. The clinical and microbiological data of all consecutive patients with $\geq 1$ positive blood culture for $K$. pneumoniae strains showing non-susceptibility to tigecycline (minimum inhibitory concentration $(\mathrm{MIC})>2 \mathrm{mg} / \mathrm{L}$ ) were collected. For patients with more than 2 positive blood cultures, only the first blood culture was included. Patients $<20$ years of age and those with incomplete medical records were excluded. All clinical isolates were taken as part of standard care. The study protocol was approved by the Review Board of Taipei Veterans General Hospital (Taipei, Taiwan).

\section{Strain identification and antimicrobial susceptibility test}

Identification and antimicrobial susceptibility of K. pneumoniae were determined using the Vitek2 system (bioMérieux, Marcy-l'Etoile, France). Antimicrobial susceptibility was interpreted according to the guidelines of the Clinical and Laboratory Standards Institute (CLSI) [15]. K. pneumoniae strains showing non-susceptibility to tigecycline ( $\mathrm{MIC}>2 \mathrm{mg} / \mathrm{L})$ according to the Vitek2 system were tested further by the E-test method (AB Biodisk, Solna, Sweden) according to manufacturer instructions. Breakpoints for tigecycline were those set by the US Food and Drug Administration (FDA) (2 and $8 \mathrm{mg} / \mathrm{L}$ for susceptible and resistant, respectively) [16]. Escherichia coli ATCC 25922, E. coli ATCC 35218 and Pseudomonas aeruginosa ATCC 27853 were used as control strains in the susceptibility assays.

\section{Molecular characterization of $\beta$-lactamases}

All tigecycline non-susceptible strains were tested by polymerase chain reaction (PCR) amplification and DNA sequencing for various carbapenemase genes (encoding class-B families IMP, VIM, NDM-1, GIM, SPM and SIM; class-A families NMC, IMI, SME, KPC and GES; and class-D family OXA-48), plasmid-borne ampC-like genes (encoding CMY, DHA and ACT) and ESBL genes (encoding
CTX-M, TEM, and SHV) using methodology and primers described previously [17].

\section{Capsular genotype}

To determine the capsular genotypes of $K$. pneumoniae, we undertook cps genotyping by the PCR detection of K serotype-specific alleles at $w z y$ and $w z x$ loci, including serotypes $\mathrm{K} 1$, K2, K5, K20, K54, and $\mathrm{K} 57$, as described previously [18]. These capsular serotypes are regarded as having the highest association with an invasive disease or pathogenicity [19].

\section{Pulsed-field gel electrophoresis (PFGE)}

Total DNA was prepared and PFGE was conducted as described previously [17]. Restriction enzyme XbaI (New England BioLabs, Ipswich, MA, USA) was used at the temperature suggested by the manufacturer. Restriction fragments were separated by PFGE in 1\% agarose gel (Bio-Rad, Hercules, CA, USA) in 0.56 TBE buffer (45 mM Tris, $45 \mathrm{mM}$ boric acid, $1.0 \mathrm{mM}$ ethylenediamine tetraacetic acid (EDTA); pH 8.0) for $22 \mathrm{~h}$ at $200 \mathrm{~V}$ and $14^{\circ} \mathrm{C}$ and with ramp times of 2-40 s using CHEF Mapper apparatus (Bio-Rad). Gels were then stained with ethidium bromide and photographed under ultraviolet light. The Dice coefficient was used to calculate similarities. The unweighted pair-group method with arithmetic mean (UPGMA) was used for cluster analyses by BioNumerics ver5.10 (Applied Maths, Austin, TX, USA).

\section{Data collection}

Medical records were reviewed to extract pertinent information, including demographic characteristics; comorbid conditions and the Charlson Comorbidity Index [20], duration of hospital stay, duration of therapy with individual antimicrobial drugs, antimicrobial therapy administrated before the onset of bacteremia, and a ventilator, central venous catheter, or a Foley catheter at the time of bacteremia onset. The onset of bacteremia was defined as the day the blood culture that eventually yielded $K$. pneumoniae was obtained. Bacteremia was defined as nosocomialacquired if the index blood culture was collected $>48 \mathrm{~h}$ after hospital admission and no signs or symptoms of infection was noted upon hospital admission. Infections with onset $\leq 48 \mathrm{~h}$ after hospital admission were classified as healthcare-associated or community-acquired in accordance with definitions described previously [6]. Episodes of bacteremia were considered acquired in the intensive care unit (ICU) if they appeared $>48 \mathrm{~h}$ after ICU admission. Infection severity was evaluated using the Acute Physiology and Chronic Health Evaluation (APACHE) II score and Pitt Bacteremia Score described within $24 \mathrm{~h}$ before the onset of bacteremia [21,22]. Appropriate antimicrobial therapy was defined as administration of $\geq 1$ antimicrobial agent to which the causative pathogen was susceptible 
in vitro within $48 \mathrm{~h}$ after the onset of bacteremia with an approved route and dose appropriate for end-organ function. Antimicrobial therapy that did not meet this definition was considered inappropriate. The primary outcome measure was all-cause 28-day mortality after the onset of $K$. pneumoniae bacteremia.

\section{Statistical analyses}

Categorical variables are absolute numbers and their relative frequencies. Quantitative variables are mean and standard deviation (SD) if distributed normally, or as median and interquartile range (IQR) if distributed non-normally. Contingency data were analyzed by the two-tailed chi-square test or the Fisher's exact test. Continuous data were analyzed by the Student's $t$-test or the Mann-Whitney $U$ test. An exact logistic regression was used in the multivariate analyses to analyze risk factors for 28-day mortality. All factors with $\mathrm{p}<0.1$ in the univariate analyses were included in the exact logistic regression. $\mathrm{p}<0.05$ was considered as significant. Statistical analyses were carried out using SAS ver9.2 (SAS Institute Inc., Cary, NC, USA).

\section{Results}

\section{Microbiological characteristics}

During the study period, 40 patients with bacteremia due to tigecycline non-susceptible $K$. pneumoniae were identified. Thirty-six patients were finally enrolled after confirmation of susceptibility by the E-test method. The $\mathrm{MIC}_{50}$ and the $\mathrm{MIC}_{90}$ of tigecycline were 6 and $8 \mathrm{mg} / \mathrm{L}$, respectively. The tigecycline non-susceptibility rate for K. pneumoniae bacteremia during the study period was $0.05 \%$. The in vitro activities of the tested antimicrobial agents against the 36 tigecycline non-susceptible $K$. pneumoniae isolates are shown in Table 1 . Among the 36 isolates, 8 isolates were not susceptible to ertapenem (MIC $\geq 1 \mu \mathrm{g} / \mathrm{mL}$ ). Of the 8 isolates not susceptible to ertapenem, 5 isolates were resistant to ertapenem ( $\mathrm{MIC} \geq 2 \mu \mathrm{g}$ / $\mathrm{mL}$ ) and two of them were not susceptible to imipenem ( $\mathrm{MIC}=2$ and $4 \mu \mathrm{g} / \mathrm{mL}$, respectively).

One pair of $K$. pneumoniae strains had indistinguishable PFGE patterns (Figure 1). According to criteria described previously, no major cluster of isolates was found among those 36 isolates.

Carbapenemase genes were not detected among any of the 36 isolates. Among the isolates producing ESBLs, bla $a_{\mathrm{CTX}-\mathrm{M}}$ was detected in 6 isolates. Five isolates expressed CTX-M-15, and 1 isolate expressed CTX-M-14. bla $a_{\mathrm{SHV}-11}$, $b l a_{\mathrm{SHV}-12}$ and $b l a_{\mathrm{SHV}-31}$ were detected in 7,3 , and 2 isolates, respectively. With regard to AmpC-type $\beta$-lactamases, $b l a_{\mathrm{DHA}-1}$ was detected in 18 isolates, and $b l a_{\mathrm{CMY}-2}$ and bla $a_{\mathrm{ACT}-1}$ were not detected among these isolates (Table 2).

The results of capsular genotyping for the 6 genotypes (K1, K2, K5, K20, K54 and K57) showed that 2 isolates
Table 1 In vitro activities of tested antimicrobial agents against 36 tigecycline non-susecptible $K$. pneumoniae isolates

\begin{tabular}{|c|c|c|c|c|}
\hline $\begin{array}{l}\text { Antimicrobial } \\
\text { agent }\end{array}$ & $\begin{array}{l}\mathrm{MIC}^{\mathrm{a}} \text { range } \\
(\mu \mathrm{g} / \mathrm{mL})\end{array}$ & $\begin{array}{l}\mathrm{MIC}_{50}{ }^{\mathrm{b}} \\
(\mu \mathrm{g} / \mathrm{mL})\end{array}$ & $\begin{array}{l}\mathrm{MIC}_{90}{ }^{\mathrm{C}} \\
(\mu \mathrm{g} / \mathrm{mL})\end{array}$ & $\begin{array}{l}\text { No. (\%) } \\
\text { of isolates } \\
\text { susceptible }\end{array}$ \\
\hline Tigecycline & $3-32$ & 6 & 8 & \\
\hline Ciprofloxacin & $<0.25$ to $>4$ & 2 & $>4$ & $15(42)$ \\
\hline Levofloxacin & 0.5 to $>8$ & $>8$ & $>8$ & $14(39)$ \\
\hline Ampicillin-sulbactam & 8 to $>32$ & $>32$ & $>32$ & $2(6)$ \\
\hline Piperacillin-tazobactam ${ }^{*}$ & 8 to $>128$ & 32 & $>128$ & $12(48)$ \\
\hline Cefazolin & $<1$ to $>64$ & $>64$ & $>64$ & $13(36)$ \\
\hline Ceftriaxone & $<1$ to $>64$ & $<1$ & $>64$ & $21(58)$ \\
\hline Ceftazidime & $<1$ to $>64$ & 16 & $>64$ & $16(44)$ \\
\hline Cefepime & $<1$ to $>64$ & $<1$ & $>64$ & $23(64)$ \\
\hline Amikacin & $<2$ to $>64$ & $<2$ & 16 & $33(92)$ \\
\hline Gentamicin & $<1$ to $>16$ & $<1$ & $>16$ & $22(61)$ \\
\hline Ertapenem & $<0.5$ to $>8$ & $<0.5$ & 4 & $28(78)$ \\
\hline Imipenem & $<0.25$ to 4 & $<1$ & $<1$ & $34(94)$ \\
\hline
\end{tabular}

${ }^{\mathrm{a}} \mathrm{MIC}$ : minimal inhibitory concentration.

${ }^{b} \mathrm{MIC}_{50}$ : MIC for $50 \%$ of isolates.

${ }^{\mathrm{C}} \mathrm{MIC}_{90}$ : MIC for $90 \%$ of isolates.

*Total number is 25 because 11 patients did not undergo the MIC test.

belonged to capsular type $\mathrm{K} 2$, and that 1 isolate was capsular type K54. These 3 isolates did not carry ESBL or plasmid-borne ampC-like genes.

\section{Clinical characteristics}

During the study period, no obvious increasing trends or any cluster of tigecycline non-susceptible $K$. pneumoniae bacteremia was identified. There was no epidemiological relationship between the 2 patients whose isolates had indistinguishable patterns on PFGE. The usage of tigecycline was 11.89 defined daily doses per 1000 patient-days. The clinical characteristics of the patients are summarized in Table 3. Of these patients, 27 (75\%) acquired nosocomial K. pneumoniae bacteremia, and 14 patients (38.9\%) acquired $K$. pneumoniae bacteremia in the ICU. Among the 9 patients with community-onset bacteremia, 6 patients (16.7\%) were categorized as having healthcare-associated bacteremia. Most patients received antibiotics during the last 30 days. However, only 13 patients (36.1\%) received tigecycline. We investigated further the previous culturepositive data for tigecycline-susceptible $K$. pneumoniae and associated tigecycline use in the past 90 days. Nine patients with tigecycline-susceptible $K$. pneumoniae isolates were identified, and 2 patients with tigecycline-susceptible K. pneumoniae isolated within 30 days before bacteremia has been treated with tigecycline.

Pneumonia, intra-abdominal infection, and biliary-tract infection (BTI) were the common sources of bacteremia. The clinical syndromes of patients infected with the 


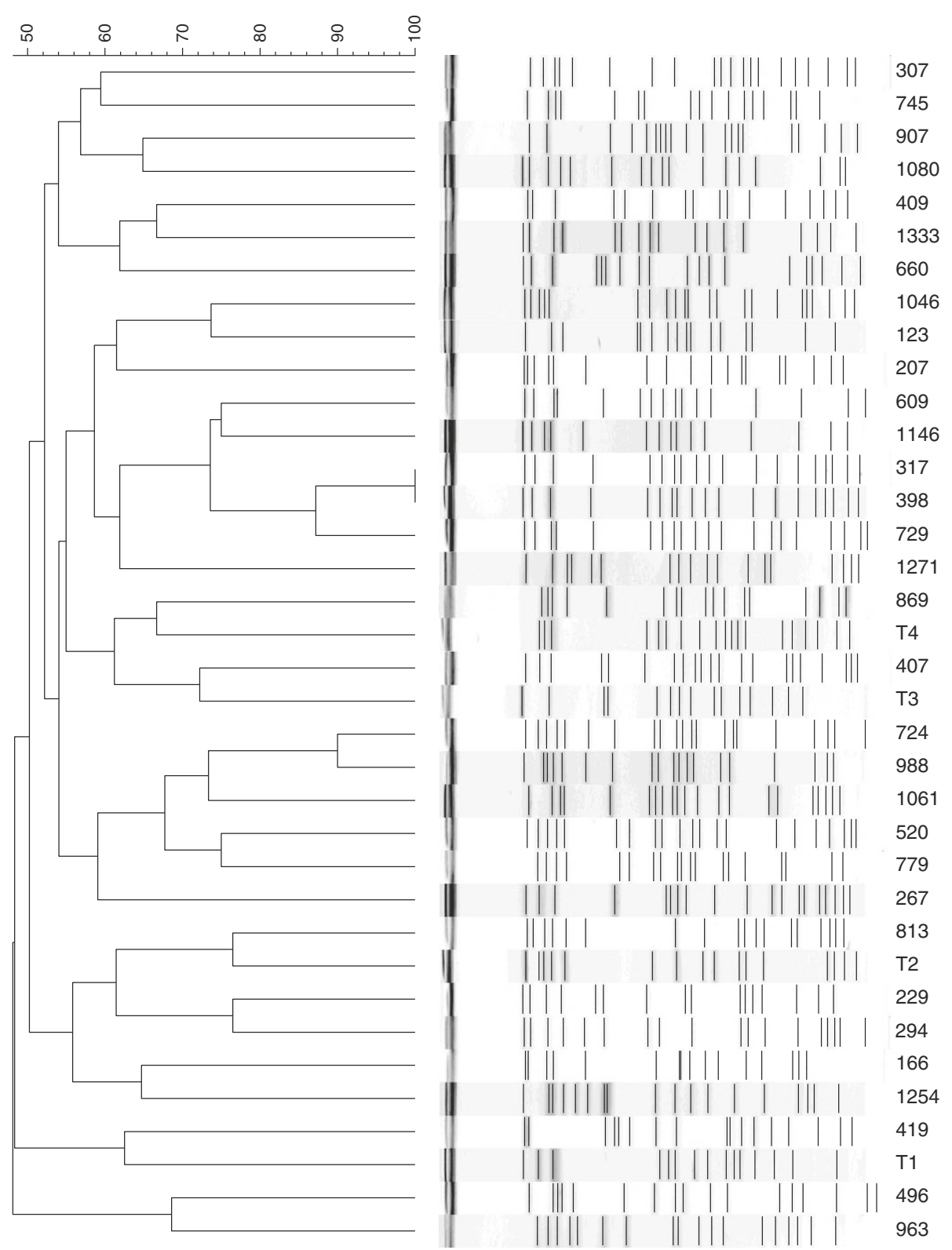

Figure 1 Pulse-field gel electrophoresis dendrogram of $36 \mathrm{~K}$. pneumoniae isolates. Most of the isolates did not have a clonal relationship. Isolates 317 and 398 showed identical patterns.

capsular type-K2 strain were pneumonia and BTI. BTI was evident in the patient infected with the capsular type K54. The treatment regimen after the sensitivity reports were available included: 13 patients received carbapenem, 11 patients received cephalosporins, 4 patients received fluoroquinolones, and 2 patients received $\beta$-lactam $/ \beta$-lactamase inhibitors. The remaining 6 patients died before the sensitivity reports were available. Among these 6 patients, 4 patients received tigecycline, and 2 patients received carbapenem empirically. The overall 28-day mortality among patients infected with tigecycline non-susceptible K. pneumoniae was $38.9 \%$.
Univariate analyses of the factors associated with 28-day mortality are shown in Table 4 . The exact logistic regression model showed that the only independent risk factor for 28-day mortality is ICU onset of tigecycline nonsusceptible Klebsiella pneumoniae bacteremia (odds ratio (OR) 13.46, 95\% confidence interval (CI) 1.35-637.17, $\mathrm{p}=0.021)$.

\section{Discussion}

We demonstrated here, for the first time, the clinical and microbiological characteristics of tigecycline nonsusceptible K. pneumoniae bacteremia in Taiwan. Most 


\begin{tabular}{|c|c|c|c|c|c|c|}
\hline Isolate & $\begin{array}{l}\text { Tigecycline } \\
\text { MIC }\end{array}$ & $\begin{array}{l}\text { Ertapenem } \\
\text { MIC }\end{array}$ & TEM & SHV & CTX-M & DHA-1 \\
\hline 1 & 3 & $<0.5$ & - & SHV-1 & - & + \\
\hline 2 & 3 & $<0.5$ & - & SHV-12 & - & + \\
\hline 3 & 3 & 8 & - & $-{ }^{a}$ & - & - \\
\hline 4 & 3 & $<0.5$ & TEM-1 & SHV-11 & - & - \\
\hline 5 & 4 & $<0.5$ & TEM-1 & SHV-11 & CTXM-15 & + \\
\hline 6 & 4 & 2 & - & SHV-1 & СTXM-15 & + \\
\hline 7 & 4 & $<0.5$ & - & SHV-12 & - & + \\
\hline 8 & 4 & $<0.5$ & - & SHV-31 & - & + \\
\hline 9 & 4 & $<0.5$ & TEM-1 & SHV-2 & - & - \\
\hline 10 & 4 & 1 & TEM-1 & SHV-1 & CTXM-15 & + \\
\hline 11 & 6 & 8 & - & SHV-2 & - & + \\
\hline 12 & 6 & 4 & - & SHV-1 & - & + \\
\hline 13 & 6 & $<0.5$ & TEM-1 & SHV-1 & CTXM-15 & + \\
\hline 14 & 6 & $<0.5$ & TEM-1 & SHV-1 & CTXM-15 & + \\
\hline 15 & 6 & $<0.5$ & TEM-1 & SHV-11 & - & + \\
\hline 16 & 6 & 1 & TEM-1 & SHV-11 & - & + \\
\hline 17 & 8 & 1 & TEM-1 & SHV-11 & CTXM-14 & + \\
\hline 18 & 8 & $<0.5$ & - & SHV-12 & - & + \\
\hline 19 & 8 & $<0.5$ & - & $-^{a}$ & - & + \\
\hline 20 & 8 & 8 & - & SHV-31 & - & - \\
\hline 21 & 16 & $<0.5$ & - & SHV-11 & - & + \\
\hline 22 & 32 & $<0.5$ & TEM-1 & SHV-11 & - & + \\
\hline
\end{tabular}

${ }^{\mathrm{a}}$ The result showed OPK-B.

patients had nosocomial bacteremia, and the overall 28 -day mortality was $38.9 \%$. The $\mathrm{MIC}_{50}$ and $\mathrm{MIC}_{90}$ of tigecycline were 6 and $8 \mathrm{mg} / \mathrm{L}$, respectively. Among all isolates, 18 were ESBL producers, and DHA-1 was detected in 18 isolates. No carbapenemase genes were detected among all 36 isolates. According to the results of PFGE, most isolates were epidemiologically unrelated.

Interpretive criteria for the in vitro susceptibility testing of tigecycline are based on the breakpoints approved by the FDA or on those published by the European Committee on Antimicrobial Susceptibility Testing. The CLSI has not released tentative or approved guidelines. Differences in susceptibility results according to the MIC interpretive criteria of the two guidelines as well as the testing methods used have been emphasized in several reports [23]. One recent study questioned the performance of the Vitek2 automated system to reliably determine the susceptibility of Enterobacteriaceae species other than $E$. coli to tigecycline [23]. Moreover, it has been reported that the E-test shows the best correlation with the broth microdilution method, and exhibits lowest error rates as well as highest essential agreement and categorical agreement [24]. In general, the
Vitek2 automated system produces higher MIC values than E-test doses, which can potentially result in false resistance findings using the former approach [24]. E-test susceptibility results obtained using the FDA criteria could be considered as a reliable method for tigecycline testing, as shown previously [25]. In the current study, isolates reported as being non-susceptible to tigecycline by the Vitek2 system were confirmed with the E-test method to avoid the misclassification of the results.

Common sources of bacteremia in our study include infections of the lower respiratory tract, BTI, and intraabdominal infections. One report suggested that clinicians should be aware of the potential risk for treatment-induced tigecycline resistance, especially if urine is the main source of bacteria [22]. In contrast to that report, we identified only 1 case with a urinary-tract infection. Physicians should recognize that the emergence of tigecycline non-susceptible K. pneumoniae bacteremia can be associated with various syndromes.

The mechanisms of resistance mediating the effectiveness of tigecycline have been attributed to RNDtype transporters and other efflux pumps [10]. Analyses of clinical studies suggest that long-term tigecycline monotherapy may carry a higher risk for developing tigecycline resistance [10]. One recent study investigating non-susceptibility in $K$. pneumoniae and E. coli isolates causing neonatal septicemia found increasing tigecycline MIC values from 2007 to 2010. This increase cannot be attributed to the use of tigecycline because of the restricted use of this antibiotic in their hospital. The author suggested that the elevated MIC of tigecycline might be attributed indirectly to the use of other antibiotics that are also effluxed through the AcrAB-TolC pump [14]. In the current study, although most patients received one or more antibiotics in the past 30 days, only $36.1 \%$ of subjects were treated with tigecycline in the past 30 days. Further study to analyze the risk factors for tigecycline non-susceptible $K$. pneumoniae infection is necessary.

In the present study, PFGE analyses revealed that tigecycline non-susceptible $K$. pneumoniae isolates causing bacteremia were not clonal in Taiwan. This implies that the acquisition of tigecycline non-susceptibility in invasive $K$. pneumoniae isolates was most likely through independent mechanisms. Such non-clonal occurrence of tigecycline non-susceptibility in invasive $K$. pneumoniae isolates suggests that tigecycline resistance may be due to selective pressure, including increasing, inappropriate and inadequate use of antibiotics. Antibiotic stewardship will be essential for helping us understand whether this holds true in real-life situations.

The 28-day mortality among patients with tigecycline non-susceptible K. pneumoniae bacteremia was $38.9 \%$. The case mortality of $K$. pneumoniae bacteremia has been found to be $\approx 20 \%$ [1]. With respect to drug-resistant 
Table 3 Characteristics of 36 adult patients with tigecycline non-susecptible $K$. pneumoniae bacteremia

\begin{tabular}{l} 
Characteristic \\
\hline Age (years, median, IQR) \\
Male sex \\
Underlying disease \\
Immunosuppression ${ }^{\text {a }}$ \\
Diabetes mellitus \\
Chronic obstructive lung disease \\
Cerebral vascular disease \\
Chronic kidney disease, stage $=4$ or 5 \\
Hemodialysis \\
Malignancy \\
Hematological malignancy \\
Solid tumor \\
Liver cirrhosis
\end{tabular}

Charlson comorbidity score (median, IQR)

Community-acquired infections

Healthcare-associated infections ${ }^{\text {b }}$

Nosocomial infections ${ }^{b}$

ICU

Medical ward

Surgical ward

Polymicrobial infection

Antibiotic exposure in the past 30 days, $\geq 3$ days

Any
Tigecycline
Glycopeptides
$\beta$-lactams plus $\beta$-lactamase inhibitors
First- and second-generation cephalosporins
Third- or fourth-generation cephalosporins
Carbapenem
Fluoroquinolones
Metronidazole

Ward, duration of stay and devices at the time of bacteremia

Acquired after ICU >48 h

Days of hospitalization before bacteremia (median, IQR)

Urinary catheter

Central venous catheter

Mechanical ventilation

Pitt bacteremia score (mean \pm SD)

APACHE II score (mean \pm SD)

Infection sources and clinical syndrome

Pneumonia

$13(36.1)$

11 (30.6)

12 (33.3)

11 (30.6)

$10(27.8)$

9 (25)

8 (22.2)

9 (25)

14 (38.9)

$48,18-82$

17 (47.2)

23 (63.9)

18 (50)

$4 \pm 4$

$23 \pm 10$

$10(27.8)$
Table 3 Characteristics of $\mathbf{3 6}$ adult patients with tigecycline non-susecptible $K$. pneumoniae bacteremia (Continued)

\begin{tabular}{ll}
\hline $\begin{array}{l}\text { Intra-abdominal infection other than } \\
\text { biliary-tract infection }\end{array}$ & $5(13.9)$ \\
Biliary-tract infection & $7(19.4)$ \\
Skin and soft-tissue infection & $2(5.6)$ \\
Catheter infection & $1(2.8)$ \\
Urinary-tract infection & $1(2.8)$ \\
Unknown primary focus & $10(27.8)$ \\
Appropriate antibiotic treatment & $17(47.2)$ \\
Outcome & \\
Presentation with septic shock & $19(52.8)$ \\
Requiring admission to ICU after bacteremia & $19(52.8)$ \\
In-hospital death & $17(47.2)$ \\
28-day mortality & $14(38.9)$
\end{tabular}

IQR, interquartile range; ICU, intensive care unit; APACHE, Acute Physiology and Chronic Health Evaluation; SD, standard deviation.

${ }^{a}$ Immunosuppression includes patients who underwent solid-organ

transplantation, corticosteroid therapy, and immunosuppression therapy.

${ }^{b}$ Bacteremia was defined to be nosocomial-acquired if the index blood culture was collected $>48 \mathrm{~h}$ after hospital admission and no signs or symptoms of infection were noted at hospital admission. Bacteremia with onset $\leq 48 \mathrm{~h}$ after hospital admission was classified as healthcare-associated infection if patients met any of the following criteria: having received intravenous therapy at home or in an outpatient clinic during the past 30 days; having received renal dialysis in a hospital or clinic during the past 30 days; having hospitalized for 2 or more days during the past 90 days; or having resided in a nursing home or long-term care facility.

$K$.pneumoniae bacteremia, the mortality in those with carbapenem-resistant $K$. pneumoniae bacteremia is $\approx 40-$ $50 \%[21,26-28]$, and $\approx 20-30 \%$ in ESBL-producing $K$. pneumoniae bacteremia [21,22]. The present report is the first to point out high mortality among patients with tigecycline non-susceptible $K$. pneumoniae bacteremia in Taiwan. One recent study found that most $(75 \%)$ of the tigecycline resistant K. pneumoniae were Klebsiella pneumoniae carbapenemase producers [29]. Surprisingly, no carbapenemase-producing $K$. pneumoniae were identified in the current study. The emergence of tigecycline nonsusceptible K. pneumoniae bacteremia, in addition to carbapenem resistance, may become an imminent issue in the foreseeable future. We also found that the ICU onset of tigecycline non-susceptible $K$. pneumoniae bacteremia was an independent risk factor for 28-day mortality. Resistance to tigecycline could be simply a marker of disease severity as shown in infections caused by other resistant microorganisms. In addition, we identified 3 isolates belonging to the capsular serotypes $\mathrm{K} 2$ and $\mathrm{K} 54$, which are thought to be associated most closely with an invasive disease and/or pathogenicity. The MDR K. pneumonaie strains rarely belonged to the virulent capsular types (K1, K2, K5, K20, K54, and K57). It would be of interest to study the relationship between these capsular types and tigecycline non-susceptible strains. 
Table 4 Risk factors for 28-day mortality among 36 patients infected by tigecycline non-susecptible $K$. pneumoniae bacteremia

\begin{tabular}{|c|c|c|c|c|}
\hline & $\begin{array}{l}\text { Survivors } \\
(\mathrm{n}=22), \mathrm{n}(\%)\end{array}$ & $\begin{array}{l}\text { Non-survivors } \\
(\mathrm{n}=14), \mathrm{n}(\%)\end{array}$ & $\mathbf{p}$ & Adjustedp $^{a}$ \\
\hline Age (years, median, IQR) & $76.5,69.3-84.3$ & $71,60.3-79$ & 0.175 & \\
\hline Male sex & $17(77.3)$ & $10(71.4)$ & 0.988 & \\
\hline \multicolumn{5}{|l|}{ Underlying disease } \\
\hline Immunosuppression $^{\text {b }}$ & $5(22.7)$ & $4(28.6)$ & 0.988 & \\
\hline Diabetes mellitus & $9(40.9)$ & $9(64.3)$ & 0.305 & \\
\hline Chronic obstructive lung disease & $2(9.1)$ & $3(21.4)$ & 0.574 & \\
\hline Hemodialysis & $5(22.7)$ & $6(42.9)$ & 0.364 & \\
\hline Malignancy & $7(31.8)$ & $7(50)$ & 0.458 & \\
\hline Liver cirrhosis & $2(9.1)$ & $1(7.1)$ & 1.000 & \\
\hline Cerebral vascular disease & $6(27.3)$ & $4(28.6)$ & 1.000 & \\
\hline Charlson comorbidity score (median, IQR) & $3,2-5$ & $4,3-8.3$ & 0.171 & \\
\hline Pitt bacteremia score (mean \pm SD) & $3.3 \pm 3.1$ & $5.9 \pm 3.7$ & 0.037 & 0.847 \\
\hline APACHE II score (mean \pm SD) & $20.1 \pm 0.6$ & $27.6 \pm 11.0$ & 0.016 & 0.958 \\
\hline Acquired after ICU $>48 \mathrm{~h}$ & $3(13.6)$ & $11(78.6)$ & $<0.001$ & 0.021 \\
\hline \multicolumn{5}{|l|}{ Infection sources and clinical syndrome } \\
\hline Pneumonia & $3(13.6)$ & $7(50)$ & 0.047 & 0.569 \\
\hline $\begin{array}{l}\text { Intra-abdominal infection other than } \\
\text { biliary-tract infection }\end{array}$ & $2(9.1)$ & $3(21.4)$ & 0.574 & \\
\hline Biliary-tract infection & $7(31.8)$ & $0(0)$ & & \\
\hline Unknown primary focus & $8(36.4)$ & $2(14.3)$ & 0.289 & \\
\hline Presentation with septic shock & $9(40.9)$ & $10(71.4)$ & 0.147 & \\
\hline Appropriate antibiotic treatment & $12(54.6)$ & $6(42.9)$ & 0.733 & \\
\hline Ertapenem non-susceptibility & $6(27.3)$ & $2(14.3)$ & 0.628 & \\
\hline Tigecycline MIC >4 $\mu \mathrm{g} / \mathrm{mL}$ & $10(45.5)$ & $8(57.1)$ & 0.733 & \\
\hline Tigecycline MIC >6 $\mu \mathrm{g} / \mathrm{mL}$ & $4(18.2)$ & $6(42.9)$ & 0.220 & \\
\hline ESBL-producing strain & $10(45.5)$ & $8(57.1)$ & 0.733 & \\
\hline
\end{tabular}

IQR, interquartile range; ICU, intensive care unit; APACHE, Acute Physiology and Chronic Health Evaluation; SD, standard deviation.

${ }^{a}$ All factors with $\mathrm{p}<0.1$ in univariate analyses were included in the exact logistic regression.

${ }^{\mathrm{b}}$ Immunosuppression includes patients who underwent solid-organ transplantation, corticosteroid therapy, and immunosuppression therapy.

Our study had several limitations. The important limitations were inherent to its retrospective design and the limited number of occurrence in a single institution. Caution must be taken to interpret data from a small number of cases. In addition, our study did not include a control group to identify specific risk factors or to compare the prognostic implications. Finally, we did not investigate the possible mechanism (e.g., overexpression of different efflux pumps) among these $K$. pneumoniae isolates. Nevertheless, our study was the first to describe the characteristics of tigecycline non-susceptible $K$. pneumoniae bacteremia in Taiwan, and will provide an important foundation for further work in this emerging area of interest.

\section{Conclusion}

The high mortality of patients with tigecycline nonsusceptible $K$. pneumoniae bacteremia may indicate a critical clinical problem. The emergence of tigecycline non-susceptible K. pneumoniae bacteremia in our hospital merits further studies to identify potential risk factors for its development.

\section{Abbreviations}

BTI: Biliary-tract infection; CLSI: Clinical and laboratory standards institute; ESBL: Extended-spectrum $\beta$-lactamases; PFGE: Pulsed-field gel electrophoresis; FDA: Food and drug administration.

\section{Competing interests}

The authors declare that they have no competing interests.

Authors' contributions

YTL conceived the study, and participated in its design and coordination. YTL, FDW, YJC, and YCF reviewed and collected the data. YTL analyzed and interpreted the data. YTL drafted the manuscript. FDW and CPF reviewed the manuscript. All authors approved the final manuscript. 


\section{Acknowledgments}

Some results from this study were presented orally at the 28th International Congress of Chemotherapy and Infection (ICC 2013) in Yokohama, Japan. This work was supported by grants from Taipei Veterans General Hospital (V102B-022 and V103B-016). The authors thank Ms. Chiu-Mei Yeh for her endorsement and assistance in the statistical analyses.

\section{Author details}

'Division of Infectious Disease, Department of Medicine, Taipei Veterans General Hospital, No. 201, Sec. 2, Shih-Pai Road, Taipei 112, Taiwan. ${ }^{2}$ School of Medicine, National Yang-Ming University, Taipei, Taiwan. ${ }^{3}$ Division of Microbiology, Department of Pathology and Laboratory Medicine, Taipei Veterans General Hospital, Taipei, Taiwan.

Received: 17 October 2013 Accepted: 9 December 2013 Published: 1 January 2014

\section{References}

1. Meatherall BL, Gregson D, Ross T, Pitout JDD, Laupland KB: Incidence, risk factors, and outcomes of Klebsiella pneumoniae bacteremia. Am J Med 2009, 122(9):866-873.

2. Podschun R, Ullmann U: Klebsiella spp. As nosocomial pathogens: epidemiology, taxonomy, typing methods, and pathogenicity factors. Clin Microbiol Rev 1998, 11:589-603.

3. Lin YT, Chen TL, Siu L, Hsu SF, Fung CP: Clinical and microbiological characteristics of community-acquired thoracic empyema or complicated parapneumonic effusion caused by Klebsiella pneumoniae in Taiwan. Eur J Clin Microbiol Infect Dis 2010, 29:1003-10.

4. Lin YT, Jeng YY, Chen TL, Fung CP: Bacteremic community-acquired pneumonia due to Klebsiella pneumoniae: clinical and microbiological characteristics in Taiwan, 2001-2008. BMC Infect Dis 2010, 10:307.

5. Tseng CP, Wu HS, Wu TH, Lin YT, Fung CP: Clinical characteristics and outcome of patients with community-onset Klebsiella pneumoniae bacteremia requiring intensive care. J Microbiol Immunol Infect 2013, 46:217-23.

6. Wu HS, Wang FD, Tseng CP, Wu TH, Lin YT, Fung CP: Characteristics of healthcare-associated and community-acquired Klebsiella pneumoniae bacteremia in Taiwan. J Infect 2012, 64:162-8.

7. Lin YT, Wang FD, Wu PF, Fung CP: Klebsiella pneumoniae liver abscess in diabetic patients: association of glycemic control with the clinical characteristics. BMC Infect Dis 2013, 13:56.

8. Keynan $Y$, Rubinstein $\mathrm{E}$ : The changing face of Klebsiella pneumoniae infections in the community. Int J Antimicrob Agents 2007, 30:385-9.

9. Livermore DM: Has the era of untreatable infections arrived? J Antimicrob Chemother 2009(1):i29-36.

10. Sun Y, Cai Y, Liu X, Bai N, Liang B, Wang R: The emergence of clinical resistance to tigecycline. Int J Antimicrob Agents 2013, 41:110-6.

11. Rodríguez-Avial C, Rodríguez-Avial I, Merino P, Picazo JJ: Klebsiella pneumoniae: development of a mixed population of carbapenem and tigecycline resistance during antimicrobial therapy in a kidney transplant patient. Clin Microbiol Infect 2012, 18:61-6.

12. Daphnis E, Neonakis I, Stylianou K, Maraki S: First case of resistance to tigecycline by Klebsiella pneumoniae in a European University Hospital. Indian J Med Microbiol 2011, 29(1):78-9.

13. Tsai HY, Liao CH, Cheng A, Liu CY, Huang YT, Sheng WH, Hsueh PR: Emergence of tigecycline-resistant Klebsiella pneumoniae after tigecycline therapy for complicated urinary tract infection caused by carbapenem-resistant Escherichia coli. J Infect 2012, 65:584-6.

14. Roy S, Datta S, Viswanathan R, Singh AK, Basu S: Tigecycline susceptibility in Klebsiella pneumoniae and Escherichia coli causing neonatal septicaemia (2007-10) and role of an efflux pump in tigecycline non-susceptibility. J Antimicrob Chemother 2013, 68:1036-42.

15. Clinical and Laboratory Standards Institute: Performance standards for antimicrobial susceptibility testing: twenty-second informational supplement. Wayne, PA: CLSI: Document M100-S22; 2012.

16. Pillar CM, Draghi DC, Dowzicky MJ, Sahm DF: In vitro activity of tigecycline against Gram-positive and Gram-negative pathogens as evaluated by broth microdilution and Etest. J Clin Microbiol 2008, 46:2862-7.

17. Ma L, Lu PL, Siu LK, Hsieh MH: Molecular typing and resistance mechanisms of imipenem-non-susceptible Klebsiella pneumoniae in Taiwan: results from the Taiwan surveillance of antibiotic resistance (TSAR) study, 2002-2009. J Med Microbiol 2013, 62:101-7.
18. Fang $C T$, Lai SY, Yi WC, Hsueh PR, Liu KL, Chang SC: Klebsiella pneumoniae genotype K1: an emerging pathogen that causes septic ocular or central nervous system complications from pyogenic liver abscess. Clin Infect Dis 2007, 45:284-93.

19. Turton JF, Perry C, Elgohari S, Hampton CV: PCR characterization and typing of Klebsiella pneumoniae using capsular type-specific, variable number tandem repeat and virulence gene targets. J Med Microbiol 2010, 59:541-7.

20. Charlson ME, Pompei P, Ales KL, Mackenzie CR: A new method of classifying prognostic comorbidity in longitudinal studies: development and validation. J Chronic Dis 1987, 40:373-83.

21. Ben-David D, Kordevani R, Keller N, Tal I, Marzel A, Gal-Mor O, Maor Y, Rahav G: Outcome of carbapenem resistant Klebsiella pneumoniae bloodstream infections. Clin Microbiol Infect 2012, 18:54-60.

22. Qureshi ZA, Paterson DL, Peleg AY, Adams-Haduch JM, Shutt KA, Pakstis DL, Sordillo E, Polsky B, Sandkovsky G, Bhussar MK, Doi Y: Clinical characteristics of bacteraemia caused by extended-spectrum $\beta$-lactamase-producing Enterobacteriaceae in the era of CTX-M-type and KPC-type $\beta$-lactamases. Clin Microbiol Infect 2012, 18:887-93.

23. Huang TD, Berhin C, Bogaerts P, Glupczynski Y: In vitro susceptibility of multidrug-resistant Enterobacteriaceae clinical isolates to tigecycline. J Antimicrob Chemother 2012, 67:2696-9.

24. Zarkotou O, Pournaras S, Altouvas G, Pitiriga V, Tziraki M, Mamali V, Themeli-Digalaki K, Tsakris A: Comparative evaluation of tigecycline susceptibility testing methods for expanded-spectrum cephalosporin- and carbapenem-resistant Gram-negative pathogens. J Clin Microbio/ 2012, 50:3747-50

25. Bolmström A, Karlsson Å, Engelhardt A, Ho P, Petersen PJ, Bradford PA, Jones $\mathrm{CH}$ : Validation and reproducibility assessment of tigecycline MIC determinations by Etest. J Clin Microbiol 2007, 45:2474-9.

26. Qureshi ZA, Paterson DL, Potoski BA, Kilayko MC, Sandovsky G, Sordillo E, Polsky B, Adams-Haduch JM, Doi Y: Treatment outcome of bacteremia due to KPC-producing Klebsiella pneumoniae: superiority of combination antimicrobial regimens. Antimicrob Agents Chemother 2012, 56:2108-13.

27. Tumbarello M, Viale P, Viscoli C, Trecarichi EM, Tumietto F, Marchese A, Spanu T, Ambretti S, Ginocchio F, Cristini F, Losito AR, Tedeschi S, Cauda R, Bassetti M: Predictors of mortality in bloodstream infections caused by Klebsiella pneumoniae carbapenemase-producing $K$. pneumoniae: importance of combination therapy. Clin Infect Dis 2012, 55:943-50.

28. Capone A, Giannella M, Fortini D, Giordano A, Meledandri M, Ballardini M, Venditti M, Bordi E, Capozzi D, Balice MP, Tarasi A, Parisi G, Lappa A, Carattoli A, Petrosillo N, SEERBIO-GRAB network: High rate of colistin resistance among patients with carbapenem-resistant Klebsiella pneumoniae infection accounts for an excess of mortality. Clin Microbiol Infect 2013, 19:E23-E30.

29. Nigo M, Cevallos CS, Woods K, Flores VM, Francis G, Perlman DC, Revuelta M, Mildvan D, Waldron M, Gomez T, Koshy S, Jodlowski T, Riley W, Ruhe Jj: Nested case-control study of the emergence of tigecycline resistance in multidrug-resistant Klebsiella pneumoniae. Antimicrob Agents Chemother 2013, 57:5743-6.

doi:10.1186/1471-2334-14-1

Cite this article as: Lin et al:: Clinical and microbiological characteristics of tigecycline non-susceptible Klebsiella pneumoniae bacteremia in Taiwan. BMC Infectious Diseases 2014 14:1.

\section{Submit your next manuscript to BioMed Central and take full advantage of:}

- Convenient online submission

- Thorough peer review

- No space constraints or color figure charges

- Immediate publication on acceptance

- Inclusion in PubMed, CAS, Scopus and Google Scholar

- Research which is freely available for redistribution 